\title{
Increased serum bile acid level is associated with high-risk coronary artery plaques in an asymptomatic population detected by coronary computed tomography angiography
}

\author{
Bu-Chun Zhang", Jun-Hong Chen", Chu-Han Xiang, Ming-Yu Su, Xue-Shan Zhang, Yan-Feng Ma \\ Department of Cardiology, The Affiliated Hospital of Xuzhou Medical University, Xuzhou 221000, China \\ Contributions: (I) Conception and design: BC Zhang, JH Chen, YF Ma; (II) Administrative support: MY Su; (III) Provision of study materials or \\ patients: XS Zhang; (IV) Collection and assembly of data: CH Xiang, XS Zhang; (V) Data analysis and interpretation: BC Zhang; (VI) Manuscript \\ writing: All authors; (VII) Final approval of manuscript: All authors. \\ \#These authors contributed equally to this work. \\ Correspondence to: Bu-Chun Zhang, Associate professor, MD, PhD; Yan-Feng Ma, MD. Department of Cardiology, The Affiliated Hospital of Xuzhou \\ Medical University, 99 West Huaihai Road, Xuzhou 221002, China. Email: zhangbc138@sina.com.cn; mayanfeng8998@163.com.
}

\begin{abstract}
Background: There are limited data on the association between serum total bile acid level and coronary plaque characteristics. This study investigated the relationship between serum total bile acid level and the severity of coronary stenosis and coronary plaque features in an asymptomatic population using coronary computed tomography angiography (CTA).

Methods: A total of 1,137 consecutive participants with no known coronary artery disease (CAD) undergoing CTA as part of a general routine health evaluation were recruited. Serum total bile acid level and clinical parameters were assayed. Coronary stenosis and high-risk plaques features (napkin-ring sign, low-attenuation plaque, spotty calcification, positive remodelling) were evaluated. Associations between serum total bile acid concentration and high-risk coronary plaques was tested through univariate and multivariate analyses.

Results: A total of 101 high-risk coronary plaques subjects and 93 controls were eligible for study inclusion. The severity of coronary artery stenosis and high-risk coronary plaques increased with serum total bile acid level quartiles (all $\mathrm{P}<0.001$ ). The independent predictor of high-risk coronary plaques in multivariate analysis was serum total bile acid level $(\mathrm{P}<0.001)$. Receiver operating characteristic (ROC) confirmed that serum total bile acid concentration significantly differentiated high-risk coronary plaques [the area under the curve (AUC) $=0.876 ; \mathrm{P}<0.001$, with a sensitivity of $87.13 \%$ and a specificity of $86.02 \%$ ].

Conclusions: Higher serum total bile acid level was associated with the severity of coronary artery stenosis and high-risk coronary artery plaques detected by CTA in asymptomatic populations.
\end{abstract}

Keywords: Circulating bile acid; atherosclerosis; coronary computed tomography angiography (CTA); plaque

Submitted Aug 15, 2019. Accepted for publication Nov 12, 2019.

doi: $10.21037 /$ jtd.2019.12.16

View this article at: http://dx.doi.org/10.21037/jtd.2019.12.16

\section{Introduction}

Acute cardiac events often develop suddenly without any premonitory symptoms; therefore, it is very important to identify high-risk coronary plaques in asymptomatic patients (1). Several studies have demonstrated that coronary computed tomography angiography (CTA) is a rapid and reliable tool in quantifying the morphology of coronary atherosclerotic plaques compared with the conventional coronary angiography (2-4). In addition, coronary CTA also with considerable accuracy to detect ruptured plaque consistent with intravascular ultrasound (IVUS) and optical coherence tomography (OCT) $(5,6)$. Therefore, coronary CTA provides detailed information about plaque morphology and composition, and may 
become the main diagnostic imaging for non-invasive plaque characterization. Additionally, multiple studies have shown that circulating biomarkers of lipid metabolism and inflammation could predict high-risk coronary plaques (7). Therefore, CTA examination combined with screening novel circulating biomarkers for vulnerable coronary plaques will improve diagnostic accuracy for suspected coronary artery disease $(\mathrm{CAD})$ patients.

Bile acids are a large family of molecules that have a steroidal structure and are synthesized in the liver and are the major component in bile (8). Bile acid has long been recognized as an important factor in regulating lipid and glucose metabolism which is an important step in the progression of atherosclerosis. Bile acid have different types, recent human studies have revealed the association between serum total bile acid level and cardiovascular diseases or metabolic syndrome (9-11). Nonetheless, it remains unclear whether bile acid predicts the vulnerable plaques in asymptomatic populations.

In this study, we aimed to evaluate the relationship of the serum total bile acid level and high-risk coronary plaques detected by CTA among adult individuals undergoing routine health examination.

\section{Methods}

\section{Study populations}

All participants aged $>18$ years who underwent CTA using 64-slice scanner for general routine health evaluation in the Affiliated Hospital of Xuzhou Medical University between January 2015 and December 2017 were screened. We excluded individuals with (I) acute chest pain; (II) a previous history of angina or myocardial infarction; (III) previous coronary artery revascularization; (IV) renal insufficiency (creatinine $>1.5 \mathrm{mg} / \mathrm{dL}$ ); (V) with other heart disease or malignant tumours; (VI) hepatic disorder.

\section{Clinical study data collection}

Traditional CAD risk factors and laboratory parameters were retrospectively collected from patient's electronic health record in our hospital database by two different trained personnel. CAD risk factors (age, gender, hypertension, hyperlipidemia, diabetes, and smoking) were queried.

Arterial hypertension was defined as a history of high blood pressure or treatment with antihypertensive medications. Diabetes mellitus was defined by a fasting plasma glucose concentration $\geq 7.0 \mathrm{mmol} / \mathrm{L}$ or by a history diagnosis of diabetes. Dyslipidemia was manifested by elevation of the total cholesterol, the low-density lipoprotein (LDL) cholesterol and the triglyceride concentrations or patients who received lipid-lowering drugs. Smoking was defined as "current" or smoking cessation within the past 6 months.

\section{Coronary CTA analysis}

Coronary CTA images were acquired using a 256-slice multidetector CT scanner (Philips, The Netherlands). The CTA analysis was conducted by using the method of the Society of Cardiovascular Computed Tomography (12). Analysis of the CTA images was performed independently by two experienced cardiovascular imagers who were blind to the participants' information. High-risk coronary plaque features (napkin-ring sign, low-attenuation plaque, spotty calcification, positive remodelling) in the coronary lesions were evaluated according to the wellestablished criteria (13). Napkin-ring sign was defined as low-attenuation area adjacent to the coronary lumen, surrounded by higher attenuation rim $<130 \mathrm{HU}(1,14)$. Low-attenuation plaque was defined as a lesion with a CT density $\leq 30 \mathrm{HU}$ (15). Spotty calcification was defined as calcific lesions $<3 \mathrm{~mm}$ in diameter (16). Vascular positive remodelling was reported as the ratio of vessel diameter at lesion site to reference vessel $>1.05$ (5).

\section{Biochemical investigations}

Blood samples were collected in the early morning after overnight fasting. Serum total bile acid was measured with commercially available Enzyme-linked Immunosorbent Assay (ELISA) Kit according to the manufacturers' instructions. The assay range of the total bile acid ELISA was $0-12.0 \mu \mathrm{mol} / \mathrm{L}$. Meanwhile, the level of serum total cholesterol, triglyceride, low-density lipoprotein cholesterol (LDL-C), high-density lipoprotein cholesterol (HDL-C), lipoprotein (a) (Lp[a]), apolipoproteins A and B (apo-A, apo-B), high-sensitivity C-reactive protein (hs-CRP) and uric acid were measured by enzymatic methods in routine hospital laboratory.

\section{Statistical analysis}

Continuous data were presented as mean \pm standard 
Table 1 Characteristics of the patients

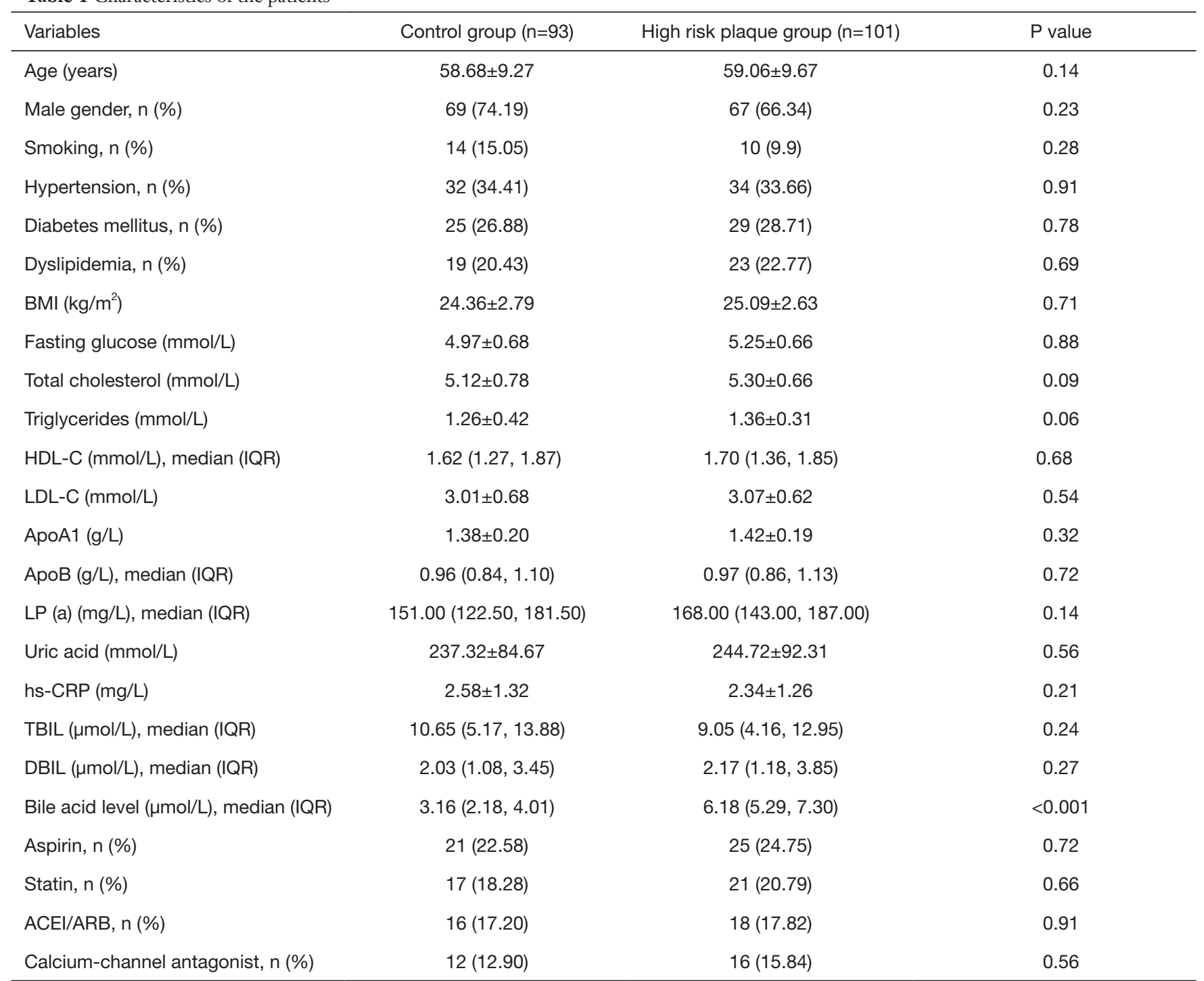

BMI, body mass index; HDL-C, high-density lipoprotein cholesterol; LDL-C, low-density lipoprotein cholesterol; hs-CRP, hypersensitive C-reactive protein; TBIL, total bilirubin; DBIL, direct bilirubin; ACEI, angiotensin-converting enzyme inhibitor; ARB, angiotensin receptor blocker; IQR, interquartile range.

deviation or as median (interquartile range, IQR). Categorical variables were given as numbers and percentages. Differences were analyzed using the Pearson chi-square or Fisher's exact (for categorical variables) and Student's $t$-test or the Mann-Whitney U test (for continuous variables).Univariable and multivariable logistic regression analyses were performed to identify the factors independently associated with high-risk plaques. Receiver operating characteristic (ROC) curve was performed to investigate the value of serum total bile acid level for high- risk coronary plaques. Statistical analyses were calculated using SPSS25.0 (IBM, Chicago, IL) and MedCalc statistical software11.5 (MedCalc Software bvba, Ostend, Belgium), respectively. $\mathrm{P}$ value $<0.05$ indicated statistical significance.

\section{Results}

A total of 1,137 participants undergoing CTA were considered for eligibility. Of these, 129 participants were excluded from analysis, including 57 subjects poor image 


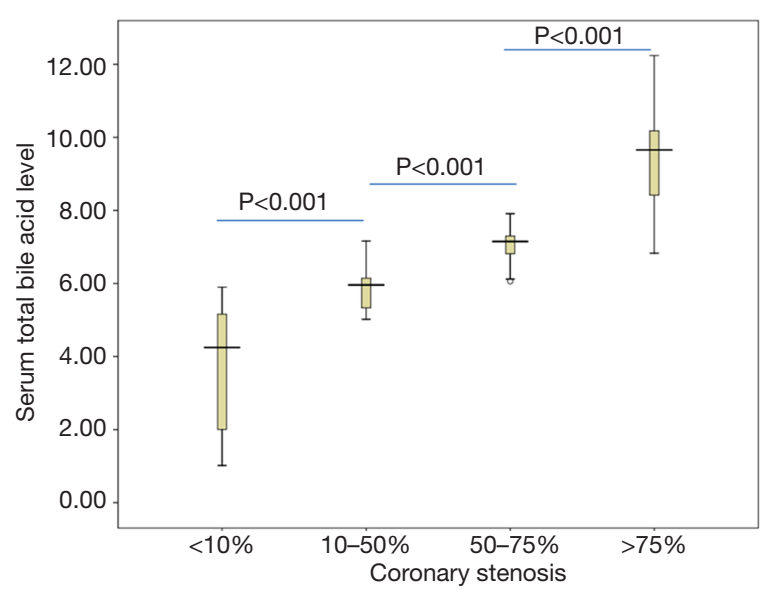

Figure 1 Relationship between the serum total bile acid level and the severity of coronary artery stenosis.

quality owing to severe calcifications or respiratory artifacts; and 72 insufficient medical records. Therefore, 1,005 participants were finally analyzed in our retrospective study according to the premised conditions. Among them, 101 asymptomatic patients with high-risk coronary artery plaques. The matched controls were derived from another 93 subjects without any coronary artery plaques in the same center at the same period.

\section{Baseline characteristics}

The clinical and biochemical characteristics of the study participants are presented in Table 1 . No significant difference was found in age, gender, smoking, hyperlipidemia, hypertension, body mass index (BMI), fasting blood-glucose, lipid profile, uric acid, and total bilirubin, direct bilirubin, hs-CRP, as well as medications between the high-risk coronary plaques group and control group.

\section{High-risk plaque distribution analysis}

Plaque analysis derived from CTA showed the prevalence of high-risk plaque features in the 101 asymptomatic patients (high-risk plaque group) was as follows: coronary plaque positive remodelling was mainly found $(n=42 ; 41.58 \%)$, followed by low-attenuation plaque ( $\mathrm{n}=30 ; 29.70 \%)$, spotty calcifications $(\mathrm{n}=25 ; 24.75 \%)$, and napkin-ring sign $(\mathrm{n}=4$; $3.96 \%)$.

\section{Association between serum total bile acid level and high- risk coronary artery plaques and coronary artery stenosis}

Subjects with high-risk coronary artery plaques [6.18 (IQR $5.29,7.30) \mu \mathrm{mol} / \mathrm{L}]$ had higher serum total bile acid level than in the controls $[3.16(\mathrm{IQR} 2.18,4.01) \mu \mathrm{mol} / \mathrm{L}]$, and the difference was statistically highly significant $(\mathrm{P}<0.001)$ (Table 1). In addition, the level of serum total bile acid was enhanced accompanied by increasing the severe coronary artery stenosis, serum total bile acid level in patients with coronary stenosis $10-50 \%$, coronary stenosis $50-75 \%$, coronary stenosis $>75 \%$ were $[5.83$ (IQR 5.02, 7.16) $\mu \mathrm{mol} / \mathrm{L}]$, [7.11 (IQR 6.06, 8.28) $\mu \mathrm{mol} / \mathrm{L}]$ and [9.41 (IQR 6.83, 12.24) $\mu \mathrm{mol} / \mathrm{L}]$, compared with coronary stenosis $<10 \%$ patients with a value of $[3.70(\mathrm{IQR} 1.02,5.90) \mu \mathrm{mol} / \mathrm{L}]$. The differences were significant between any two groups $(\mathrm{P}<0.001)$ (Figure 1).

\section{Logistic regression model}

\section{Predictors of high-risk coronary artery plaques}

Univariate analysis revealed that $\mathrm{BMI}(\mathrm{OR}=1.605,95 \% \mathrm{CI}$ : 1.007-2.558, $\mathrm{P}=0.05)$, fasting serum glucose level $(\mathrm{OR}=$ 1.726, 95\% CI: $1.211-2.461, \mathrm{P}=0.003)$, triglyceride level $(\mathrm{OR}=1.531,95 \% \mathrm{CI}: 1.082-2.168, \mathrm{P}=0.02)$, serum total bile acid level (OR =6.996, 95\% CI: 4.124-11.869, $\mathrm{P}<0.001$ ) were all significantly associated with the presence of highrisk coronary plaques. Multivariate regression analysis further demonstrated that serum total bile acid level $(\mathrm{OR}=$ 6.854, 95\% CI: 3.948-11.901, $\mathrm{P}<0.001)$ and $\mathrm{BMI}(\mathrm{OR}=$ 1.941, 95\% CI: $1.055-3.569, \mathrm{P}=0.03$ ) were independently associated with occurrence of high-risk coronary plaques (Table 2).

The relationship between the quintiles of serum total bile acid concentration and high-risk coronary plaques with logistic regression are presented in Table 3. With the analyses were performed using the lowest serum total bile acid level group as a reference. The crude ORs (95\% CI) of high-risk coronary plaques associated with the quintiles of serum total bile acid concentration were $0.46(0.11-$ 1.98), 7.91 (2.01-12.64), $37.40(10.40-134.49)$ and 64.17 $(14.82-277.75)$ (P for trend $<0.001)$. A variety of potential confounders were considered in the final multivariate logistic model, and the adjusted ORs (95\% CI) of highrisk coronary plaques associated with the quintiles of serum total bile acid concentration were $0.54(0.12-2.43), 8.05$ (2.63-24.60), $35.31(9.69-128.68)$ and $57.76(12.82-260.19)$ 
Table 2 Univariate and multivariate logistic analysis for high-risk plaques

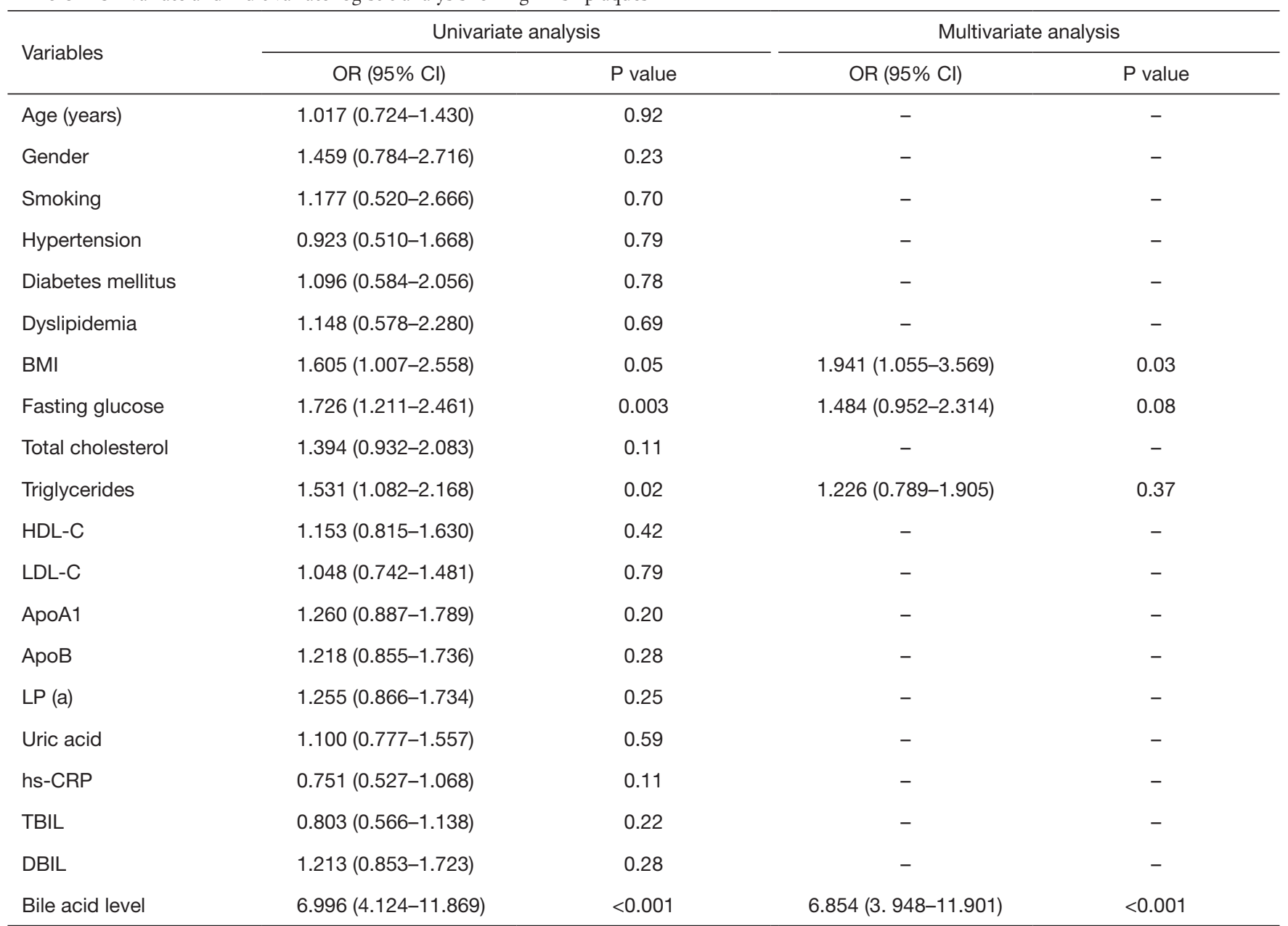

BMI, body mass index; HDL-C, high-density lipoprotein cholesterol; LDL-C, low-density lipoprotein cholesterol; hs-CRP, hypersensitive C-reactive protein; TBIL, total bilirubin; DBIL, direct bilirubin.

Table 3 The relationship between the quintiles of serum total bile acid concentration and high-risk coronary plaques

\begin{tabular}{|c|c|c|c|c|c|c|}
\hline Variables & \multicolumn{5}{|c|}{ Quintiles of serum total bile acid concentration } & $\begin{array}{l}P \text { value for } \\
\text { trend }\end{array}$ \\
\hline $\begin{array}{l}\text { Total bile acid concentration } \\
(\mu \mathrm{mol} / \mathrm{L}, \text { range })\end{array}$ & $1.02-2.71$ & $2.72-3.95$ & $3.96-5.69$ & $5.70-7.04$ & $7.05-12.24$ & \\
\hline No. of subjects & 38 & 38 & 39 & 39 & 40 & \\
\hline Model 2 & Reference & $\begin{array}{c}0.54 \\
(0.12-2.43)\end{array}$ & $\begin{array}{c}8.05 \\
(2.63-24.60)\end{array}$ & $\begin{array}{c}35.31 \\
(9.69-128.68)\end{array}$ & $\begin{array}{c}57.76 \\
(12.82-260.19)\end{array}$ & $<0.001$ \\
\hline
\end{tabular}

Model 1 crude. Model 2 was adjusted for age; gender; smoking; hypertension; body mass index (BMI); serum fasting blood-glucose level; total cholesterol; low-density lipoprotein cholesterol (LDL-C); hypersensitive C-reactive protein (hs-CRP); uric acid level. 

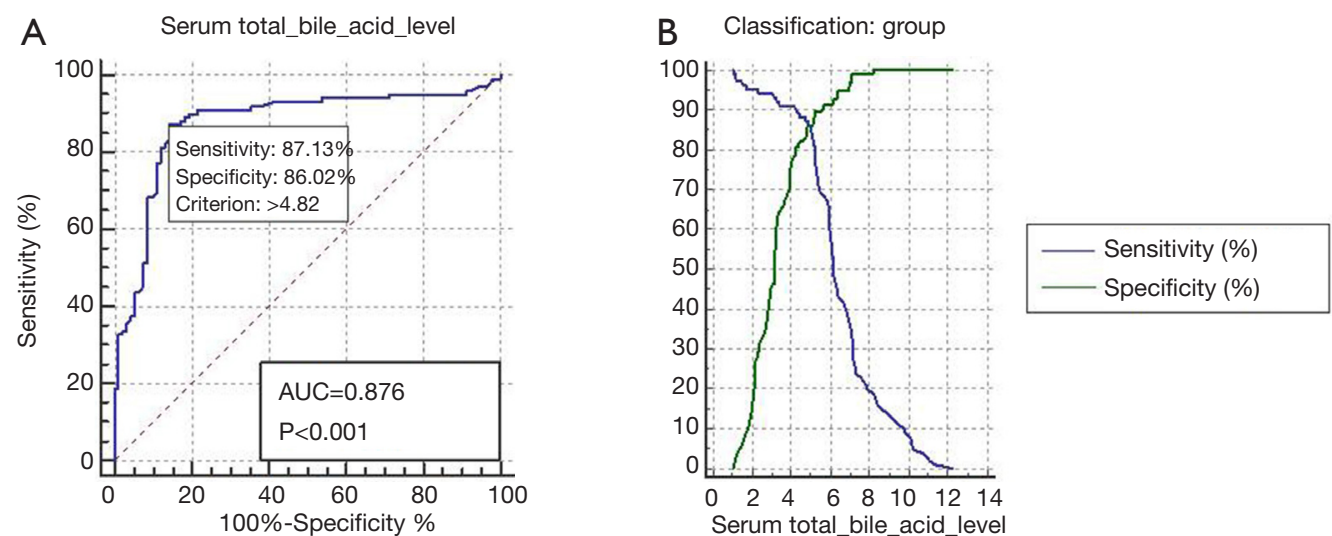

Figure 2 Receiver operating characteristic (ROC) curve of serum total bile acid level for predicting subjects with high-risk coronary plaques (AUC $=0.876,95 \%$ CI: $0.821-0.919 ; \mathrm{P}<0.001$; with a sensitivity of $87.13 \%$ and a specificity of $86.02 \%$ ).

(P for trend $<0.001)$.

\section{ROC curves}

The ROC curve was generated to detect the predictive capabilities of serum total bile acid level for high-risk coronary plaques. The area under the ROC curve (AUC) of serum total bile acid level for predicting high-risk coronary plaques among populations was 0.876 (95\% CI: $0.821-0.919 ; \mathrm{P}<0.001)$, with a sensitivity of $87.13 \%$ and a specificity of $86.02 \%$ (Figure 2).

\section{Discussion}

This study assessed the correlation of serum total bile acid level with high-risk coronary plaques in the populations with suspected but not previously diagnosed CAD. The main finding of our study was that higher serum total bile acid level was an independent predictor of high-risk coronary plaques on CTA in asymptomatic individuals after adjusting for cardiovascular risk factors.

The common risk factors for vulnerable plaques were older age, dyslipidemia, diabetes, and a history of smoking, however, this association did not persist in our study. A plausible explanation for these contrary findings maybe by differences in the study populations. Our study participants come from general population, the number of people with high-risk coronary plaques in this study was small, and the sample selection might be insufficient to represent the overall CAD disease level among adult populations.

Bile acids are the end products of cholesterol catabolism, are synthesized in the liver and affect the balance between cholesterol synthesis in the liver and cholesterol excretion. Usually, human's bodies have the ability to convert excess dietary cholesterol to bile acid under normal circumstances. However, the circulating bile acid concentrations are markedly increased under pathological conditions $(9,10)$. Apart from its important role in regulating lipid digestion and cholesterol metabolism, bile acid was also demonstrated to affect vascular inflammation and atherosclerosis (17).

The present study showed that higher circulating concentrations of total bile acid was an independent predictor of coronary artery plaque instability, with odds ratio being 6.854 (95\% CI: 3.948-11.901, $\mathrm{P}<0.001$ ). By ROC curve analysis, we found that serum total bile acid level showed its optimal cut-off value of $4.82 \mu \mathrm{mol} / \mathrm{L}$, which had a sensitivity of $87.13 \%$ and a specificity of $86.02 \%$ as serum marker for high-risk coronary plaques in this study.

Previous studies have reported a relationship between serum bile acid and cardiovascular conditions, including atherosclerosis, obesity and metabolic diseases (18). However, the exact association between serum total bile acid level and CAD population remains controversial. Steiner et al. reported that no difference in the serum bile acid level between CAD patients and healthy subjects (18), while another study by Charach et al. showed that the serum total bile acid level decreased in the CAD patients compared with controls (9). The conflicting results could be that the criteria for CAD diagnosis and the study population are different. Other studies also demonstrated that elevated serum bile acids are associated with cardiac dysfunction such as fatal arrhythmias, cardiac hypertrophy, cardiomyocyte apoptosis and abnormal heart haemodynamics (19).

The mechanisms of bile acid in atherosclerosis has not 
yet been elucidated. Several vitro studies have suggested that bile acid is toxic to different kinds of cells (20-22), and promoting endothelial cell injury by oxidative stress and pro-inflammatory responses $(23,24)$, and thereby contributing to accelerated atherosclerosis. Another in vivo study by Yamada et al. (25) using microminipigs, showed that cholic acid (main components of bile acid) could aggravate visceral obesity, atherosclerosis and nonalcoholic fatty liver disease (NAFLD) through oxidative stress signaling pathway and macrophage mobilization. On the contrary, some studies have described that bile acid receptor-farnesoid $\mathrm{X}$ receptor (FXR) may have a protective role in atherosclerosis $(26,27)$. Whether bile acid has a pathogenic effect on the development of atherosclerosis or it contributes to the regression of atherosclerosis is still unclear. Therefore, large-scale randomized studies should be conducted to investigate the role of elevation in bile acid levels in the occurrence of high-risk coronary plaques.

The limitations of the current study should be considered. First, this study did not address the cause-effect relationship between circulating bile acid level and highrisk coronary artery plaques due to a relatively small sample size and cross-sectional nature. Second, the underlying molecular mechanisms of the association between the bile acid and atherosclerotic plaques remain to be explored. Third, this is not a prospective study, the relationship between serum total bile acid level and coronary revascularization was not evaluated.

\section{Conclusions}

This study clearly shows that higher serum total bile acid level was associated with the severity of coronary artery stenosis and high-risk coronary artery plaques detected by CTA in asymptomatic populations. This association remained significant despite adjustment for other possible risk factors. Further studies are still required to elucidate the exact mechanism of bile acid in high-risk coronary plaques.

\section{Acknowledgments}

Funding: This work was supported by The National Natural Science Foundation of China (Grant number: 81670393); Jiangsu Provincial Medical Youth Talent (Grant number: QNRC2016779).

\section{Footnote}

Conflicts of Interest: The authors have no conflicts of interest to declare.

Ethical Statement: The authors are accountable for all aspects of the work in ensuring that questions related to the accuracy or integrity of any part of the work are appropriately investigated and resolved. This study was approved by the local ethics committee (reference number: XYFY2019-KL085-01), and performed according to the Helsinki Declaration. All participants signed informed consents.

\section{References}

1. Iwasaki K, Matsumoto T. Dynamic change of high-risk plaque detected by coronary computed tomographic angiography in patients with subclinical coronary artery disease. Int J Cardiovasc Imaging 2016;32:1667-73.

2. Yamamoto H, Kihara Y, Kitagawa T, et al. Coronary plaque characteristics in computed tomography and 2-year outcomes: The PREDICT study. J Cardiovasc Comput Tomogr 2018;12:436-43.

3. Oikonomou EK, Marwan M, Desai MY, et al. Noninvasive detection of coronary inflammation using computed tomography and prediction of residual cardiovascular risk (the CRISP CT study): a posthoc analysis of prospective outcome data. Lancet 2018;392:929-39.

4. Yorgun H, Canpolat U, Aytemir K, et al. Prognosis of patients with mild-moderate coronary artery stenosis detected by coronary computed tomography angiography. Int J Cardiol 2013;168:1195-200.

5. Nakazato R, Otake H, Konishi A, et al. Atherosclerotic plaque characterization by $\mathrm{CT}$ angiography for identification of high-risk coronary artery lesions: a comparison to optical coherence tomography. Eur Heart J Cardiovasc Imaging 2015;16:373-9.

6. Matsumoto H, Watanabe S, Kyo E, et al. Effect of tube potential and luminal contrast attenuation on atherosclerotic plaque attenuation by coronary CT angiography: In vivo comparison with intravascular ultrasound. J Cardiovasc Comput Tomogr 2019;13:219-25.

7. Cheng JM, Garcia-Garcia HM, de Boer SP, et al. In vivo detection of high-risk coronary plaques by radiofrequency 
intravascular ultrasound and cardiovascular outcome: results of the ATHEROREMO-IVUS study. Eur Heart J 2014;35:639-47.

8. Brandl K, Hartmann P, Jih LJ, et al. Dysregulation of serum bile acids and FGF19 in alcoholic hepatitis. J Hepatol 2018;69:396-405.

9. Charach G, Grosskopf I, Rabinovich A, et al. The association of bile acid excretion and atherosclerotic coronary artery disease. Therap Adv Gastroenterol 2011;4:95-101.

10. Charach G, Rabinovich A, Argov O, et al. The role of bile Acid excretion in atherosclerotic coronary artery disease. Int J Vasc Med 2012;2012:949672.

11. Voiosu AM, Wiese S, Voiosu TA, et al. Total bile acid levels are associated with left atrial volume and cardiac output in patients with cirrhosis. Eur J Gastroenterol Hepatol 2018;30:392-7.

12. Raff GL, Abidov A, Achenbach S, et al. SCCT guidelines for the interpretation and reporting of coronary computed tomographic angiography. J Cardiovasc Comput Tomogr 2009;3:122-36.

13. Puchner SB, Liu T, Mayrhofer T, et al. High-risk plaque detected on coronary $\mathrm{CT}$ angiography predicts acute coronary syndromes independent of significant stenosis in acute chest pain: results from the ROMICAT-II trial. J Am Coll Cardiol 2014;64:684-92.

14. Song FX, Zhou J, Zhou JJ, et al. The diagnosis of coronary plaque stability by multi-slice computed tomography coronary angiography. J Thorac Dis 2018;10:2365-76.

15. Deseive S, Straub R, Kupke M, et al. Quantification of coronary low-attenuation plaque volume for long-term prediction of cardiac events and reclassification of patients. J Cardiovasc Comput Tomogr 2018;12:118-24.

16. Finck T, Stojanovic A, Will A, et al. Long-term prognostic value of morphological plaque features on coronary computed tomography angiography. Eur Heart J Cardiovasc Imaging 2019. [Epub ahead of print].

17. Ding L, Chang M, Guo Y, et al. Trimethylamine-N-oxide (TMAO)-induced atherosclerosis is associated with bile

Cite this article as: Zhang $\mathrm{BC}$, Chen JH, Xiang $\mathrm{CH}$, Su MY, Zhang XS, Ma YF. Increased serum bile acid level is associated with high-risk coronary artery plaques in an asymptomatic population detected by coronary computed tomography angiography. J Thorac Dis 2019;11(12):5063-5070. doi: 10.21037/jtd.2019.12.16 acid metabolism. Lipids Health Dis 2018;17:286.

18. Steiner C, Othman A, Saely CH, et al. Bile acid metabolites in serum: intraindividual variation and associations with coronary heart disease, metabolic syndrome and diabetes mellitus. PLoS One 2011;6:e25006.

19. Vasavan T, Ferraro E, Ibrahim E, et al. Heart and bile acids - Clinical consequences of altered bile acid metabolism. Biochim Biophys Acta Mol Basis Dis 2018;1864:1345-55.

20. Zhang YM, Xu X, Yu Q, et al. Reversing the Cytotoxicity of Bile Acids by Supramolecular Encapsulation. J Med Chem 2017;60:3266-74.

21. Sharanek A, Burban A, Humbert L, et al. Cellular Accumulation and Toxic Effects of Bile Acids in Cyclosporine A-Treated HepaRG Hepatocytes. Toxicol Sci 2015;147:573-87.

22. Dial EJ, Dawson PA, Lichtenberger LM. In vitro evidence that phosphatidylcholine protects against indomethacin/ bile acid-induced injury to cells. Am J Physiol Gastrointest Liver Physiol 2015;308:G217-22.

23. Qin P, Tang X, Elloso MM, et al. Bile acids induce adhesion molecule expression in endothelial cells through activation of reactive oxygen species, NF-kappaB, and $\mathrm{p} 38$. Am J Physiol Heart Circ Physiol 2006;291:H741-7.

24. Parl F, Gutstein WH, D'Aguillo AF, et al. Endothelial injury. Association with elevations of serum bile acid and cholesterol concentration in biliary-obstructed rats. Atherosclerosis 1975;21:135-46.

25. Yamada S, Kawaguchi H, Yamada T, et al. Cholic Acid Enhances Visceral Adiposity, Atherosclerosis and Nonalcoholic Fatty Liver Disease in Microminipigs. J Atheroscler Thromb 2017;24:1150-66.

26. Miyazaki-Anzai S, Masuda M, Kohno S, et al. Simultaneous inhibition of FXR and TGR 5 exacerbates atherosclerotic formation. J Lipid Res 2018;59:1709-13.

27. Hageman J, Herrema H, Groen AK, et al. A role of the bile salt receptor FXR in atherosclerosis. Arterioscler Thromb Vasc Biol 2010;30:1519-28. 